\title{
New lethal disease involving type I and III collagen defect resembling geroderma osteodysplastica, De Barsy syndrome, and Ehlers-Danlos syndrome IV
}

Arja Jukkola, Saila Kauppila, Leila Risteli, Katri Vuopala, Juha Risteli, Jaakko Leisti, Leila Pajunen

\begin{abstract}
We describe the clinical findings and biochemical features of a male child suffering from a so far undescribed lethal connective tissue disorder characterised by extreme hypermobility of the joints, lax skin, cataracts, severe growth retardation, and insufficient production of type $I$ and type III procollagens. His features are compared with Ehlers-Danlos type IV, De Barsy syndrome, and geroderma osteodysplastica, as these disorders show some symptoms and signs shared with our patient.
\end{abstract}

The child died because of failure of the connective tissue structures joining the skull and the spine, leading to progressive spinal stenosis. The aortic valve was translucent and insufficient. The clinical symptoms and signs, together with histological findings, suggested a collagen defect. Studies on both skin fibroblast cultures and the patient's serum showed reduced synthesis of collagen types $I$ and III at the protein and RNA levels. The sizes of the mRNAs and newly synthesised proteins were normal, excluding gross structural abnormalities. These findings are not in accordance with any other collagen defect characterised so far.

(F Med Genet 1998;35:513-518)

Keywords: types I and III collagen defect; geroderma osteodysplastica; De Barsy syndrome; Ehlers-Danlos IV

Department of Pathology, University of Oulu, Finland

K Vuopala

Department of Clinical Chemistry, University of Oulu, Finland

J Risteli

Department of Clinical Genetics, University of Oulu, Finland

J Leisti

L Pajunen

Correspondence to: Dr Pajunen, Tahkokangas Hospital, Kiilakiventie 5

FIN-90250 Oulu, Finland

Received 8 July 1997 Revised version accepted for publication

21 November 1997 his signs and biochemical findings with previously characterised heritable disorders of connective tissue, geroderma osteodysplastica, De Barsy syndrome, and type IV EhlersDanlos syndrome, and suggest that he suffered from an unknown disease entity combining some characteristics of all of them.

\section{Case report}

The patient was born at term after an uncomplicated delivery as the first child of healthy, non-consanguineous, 27 year old, Finnish parents. The pregnancy had been followed because of mild hypertonia, oligohydramnios, and poor fetal growth. His birth weight was $2180 \mathrm{~g}$, length $43 \mathrm{~cm}$, head circumference 32.5 $\mathrm{cm}$, and Apgar scores 9/9/9. He was small for dates with thin and translucent skin, increased visibility of superficial veins, reduced subcutaneous fat tissue, and extremely loose joints and bilateral inguinal hernia, suggesting a connective tissue disorder. Severe growth retardation (below $-6 \mathrm{SD}$ ) was obvious. The infant also suffered from bilateral cataracts and dislocation of the left hip and had a club foot on the right side.

The bilateral cataracts were operated on at the age of 6 months and since then he successfully wore contact lenses. There was no response to the attempts to correct the hip dislocation and it later became bilateral. The inguinal hernias were operated on with apparently normal wound healing afterwards.

The patient's motor development was severely delayed, as he dislocated both large and small joints several times a day. He never learnt to crawl, sit, or use his hands freely for playing. He was unable to hold his head in an upright position for any length of time, and mostly spent his days sitting in a baby seat or on his parents' lap. Muscular hypotonia and brisk reflexes were observed right from the beginning, but significant clonus only became obvious with increasing age and other symptoms.

The boy spoke his first words at the age of 16 months but his speech development did not proceed further. He could understand simple, every day talk and used fewer than 10 short words, mimicking, and a couple of signs to communicate. Eye contact often made him produce peculiar grimacing faces.

By the age of 2 years 10 months he no longer had the strength to turn from back to side, and could lift his arm at maximum to a horizontal level and hold only very light objects in his hands. By that time it also became evident that even slightly upright positions were very painful for him. CT scanning of the head and cervical vertebrae showed this to be because of an anomalous atlas and dens filling the 

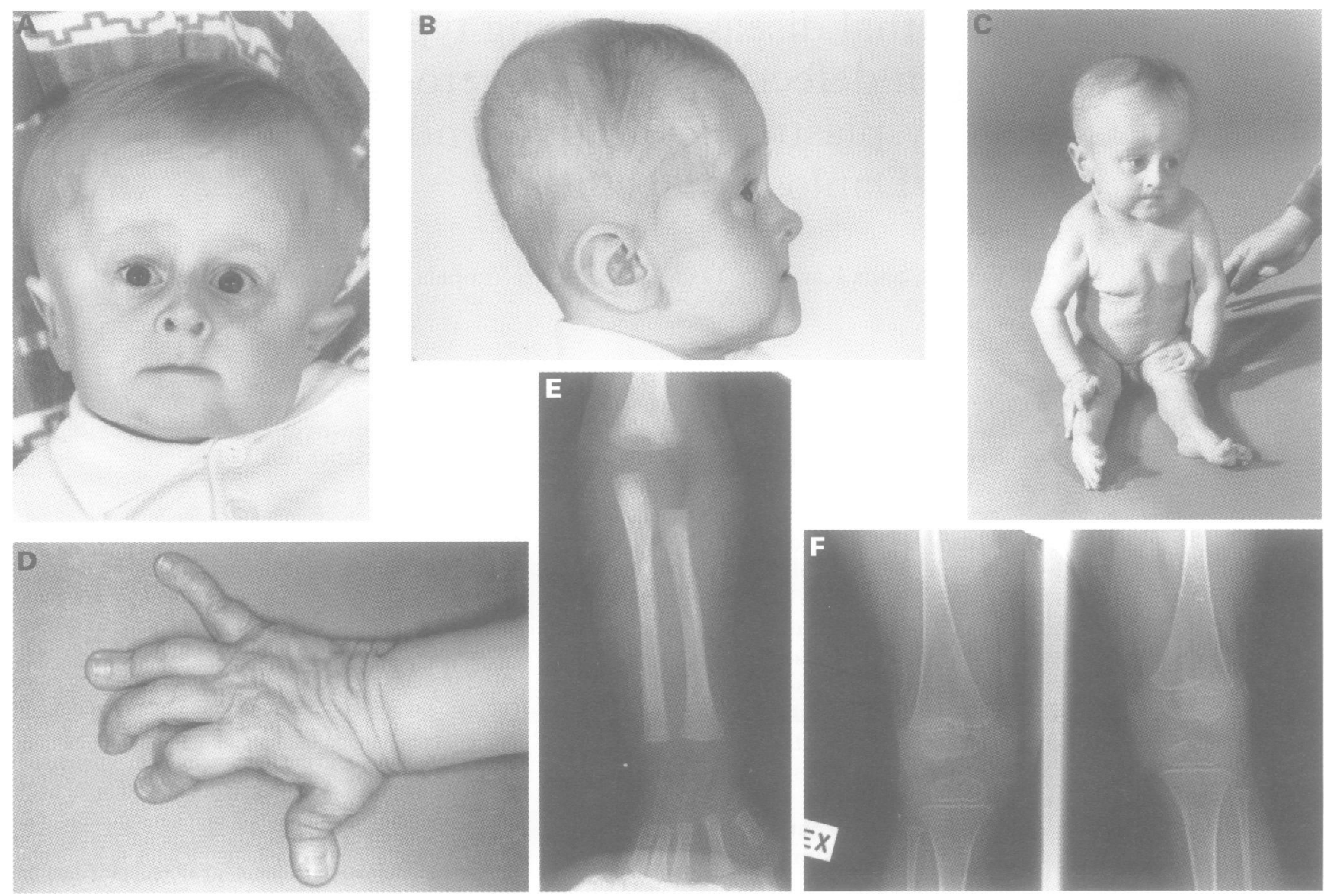

Figure 1 Pictures of the patient at 2 years of age (reproduced with permission). Anteroposterior and lateral profiles ( $A, B$ ) showing sagging cheeks, older appearance, frontal flatness, prominent nose, maxillary recession, mandibular prognathism, and visible superficial veins. Wrinkled skin, ankle deformities, and hyperextensibility of the joints $(C)$, particularly in the fingers $(D)$, are clearly visible. The radiographs of the forearm $(E, 1$ day old) and the knees $(F$, 2 years 4 months old) showed no obvious bone dysplasia, but showed the development of osteoporosis with increasing age and immobilisation.

foramen magnum so that only a $5 \mathrm{~mm}$ canal was left for the spinal cord.

As the patient's symptoms of spinal stenosis were progressive, surgical decompression was performed. He recovered well from the operation, but became increasingly restless and in pain during the next two weeks at home. The cast for neck support was changed without complications. However, within 24 hours he developed an unresponsive raised temperature, a heart rate over $200 / \mathrm{min}$, and became unconscious. His blood pressure was extremely low and did not react to medication. He died at the age of 2 years 11 months.

Table 1 Concentration of amino terminal propeptide of type III procollagen (PIIINP) and the carboxy terminal propeptide of type I procollagen (PICP) in serum samples. (Age and sex matched reference intervals in parentheses)

\begin{tabular}{lllll}
\hline & & Patient & Mother & Father \\
\hline PIIINP & $(\mu \mathrm{g} / \mathrm{l})$ & $9.6(23-43)$ & $3.6(1.7-4.2)$ & $3.2(1.7-4.2)$ \\
PICP & $(\mu \mathrm{g} / \mathrm{l})$ & $380(800-2800)$ & $190(50-170)$ & $124(38-202)$ \\
\hline
\end{tabular}

Table 2 Concentration of the amino terminal propeptide of type III procollagen (PIINP) and the carboxy terminal propeptide of type I procollagen (PICP) in the medium of cultured fibroblasts. The results are means of duplicate determinations

\begin{tabular}{lll} 
& $\begin{array}{l}\text { PIINP } \\
\text { (in the medium } \mu g / l)\end{array}$ & PICP \\
\hline $\begin{array}{l}\text { Control } \\
\text { Patient }\end{array}$ & $160(100 \%)$ & $4400(100 \%)$ \\
& $46(29 \%)$ & $700(16 \%)$ \\
\hline
\end{tabular}

\section{Clinical findings and necropsy}

The boy was examined by a clinical geneticist immediately after birth and regularly several times a year until his death. His head was large with frontal bossing and the fontanelles and cranial sutures were wide open. The mouth and nose were small, the neck was short, the left ear was slightly dysmorphic, and his small eyes had corneal clouding with bilateral cataracts, the fundus being normal. He kept his wrists in volar flexion and his long fingers showed hyperextensibility of the joints and loose, aged skin. The thumb was dug into the palm and there were bilateral simian creases (fig 1A-D).

The radiographs showed hip luxation and pes calcaneovalgus on the left and club foot on the right. The neurocranium was asymmetrical and flattened anteroposteriorly, the cranial bones were thin, and there were multiple wormian bones. The mandible was slightly hypoplastic. The right side of the atlas was attached to the base of the skull leading to mild scoliosis. No clearly recognised bone dysplasia was evident and long tubular bones were proportionally formed; osteoporosis was not prominent during the first months. However, with increasing age osteoporosis could be seen both in the tubular bones and spine (fig 1E, F).

Ultrasound examination of the abdomen was unremarkable apart from agenesis of the right kidney. He had a systolic murmur considered to be harmless, but the echocardiogram 


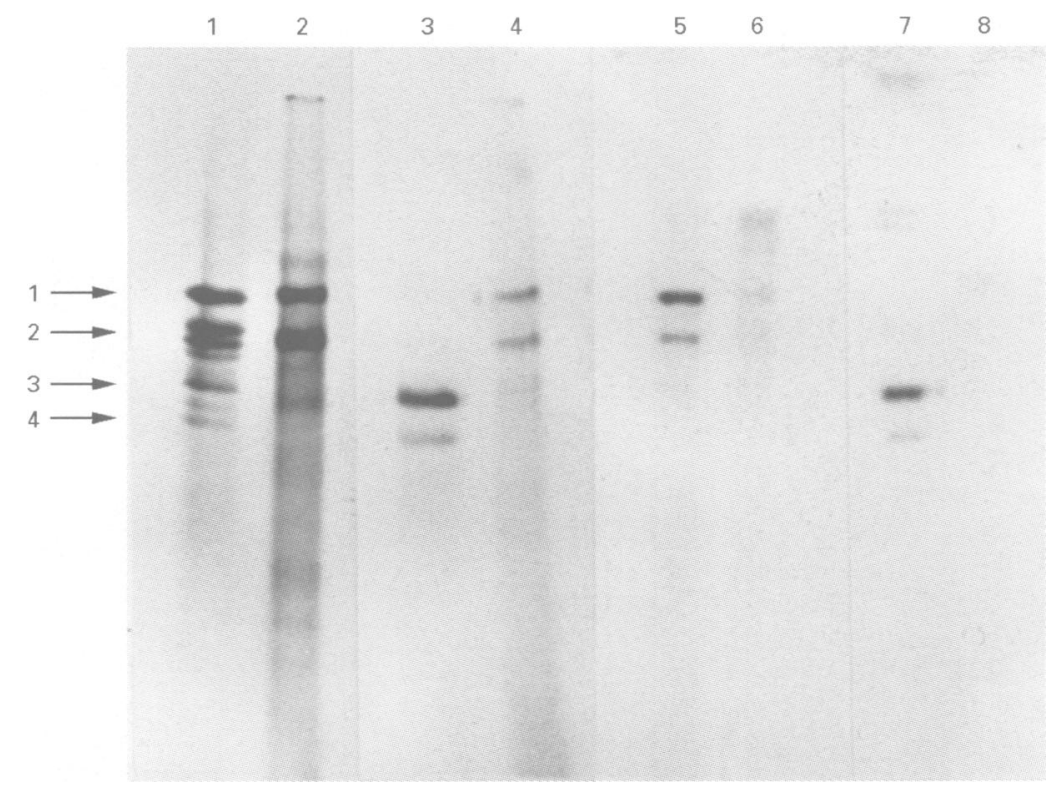

Figure 2 Sodium dodecylsulphate-polyacrylamide gel electrophoresis analysis of $(\mathrm{H} H)$ proline containing macromolecules in the medium and in the cell fraction of cultured fibroblasts after 20 hours of continuous labelling. All samples were reduced before electrophoresis. The samples were medium of control cell (lane 1), medium of patient cells (lane 2), cell fraction of control cells (lane 3), cell fraction of patient cells (lane 4), and immunoprecipitates obtained with antibodies against the amino-terminal propeptide of type III procollagen (PIIINP) in the medium of control cells (lane 5), medium of patient cells (ane 6), cell fraction of control cells (lane 7), and cell fraction of patient cells (lane 8). The molecular mass markers, indicated by arrows, were reduced proa1 (III) or proa1(I) (1), pNa1 (I) or pNa1 (III) (2), a1 (I) or a1 (III) (3), and a2(I) (4).

showed normal intracardiac anatomy and function. Blood lymphocyte karyotype, routine blood studies, and screening tests for metabolic disorders showed no pathological findings. In the CT scan taken some weeks before his death, frontotemporal cortical atrophy was seen, in addition to the spinal stenosis.

At necropsy, the aortic valve was thin, translucent, and insufficient, which was surprising as the echocardiogram a few days before death had been normal. The left side of the myocardium was hypertrophic. There were congestive changes in the lungs and liver. The central nervous system was macroscopically normally formed, but the gyri of the cerebrum were flattened. There was oedema and ischaemic changes in the frontal cortex. These findings were confirmed by a neuropathological study, in which no additional findings were found.

\section{Materials and methods}

CELL CULTURES AND PROCOLLAGEN LABELIING A skin biopsy was taken from the patient at the age of 7 months for obtaining fibroblast cultures. These cells and primary skin fibroblasts from a healthy adult control were maintained in Dulbecco's modification of Eagle's medium as described previously in detail. ${ }^{2}$ Confluent cultures were labelled overnight with $\mathrm{L}-\left(2,3,4,5-{ }^{3} \mathrm{H}\right)$ proline, and the medium and the cell layer were treated and immunoprecipitated according to published methods.

\section{IMMUNOPRECIPITATION, GEL ELECTROPHORESIS,} AND FLUOROGRAPHY

Immunoprecipitation was carried out essentially as described by Cooper et al. ${ }^{4}$ The labelled proteins were analysed by sodium dodecylsulphate-polyacrylamide gel electro- phoresis according to Laemmli, ${ }^{5}$ using $6.5 \%$ acrylamide in the separating gels. The radioactive bands were visualised by fluorography, using Kodak X-Omat autoradiographic films.

\section{RADIOIMMUNOASSAYS}

Radioimmunoassays of the equilibrium saturation type were used to determine the concentrations of the amino-terminal propeptide of human type III procollagen (PIIINP) and the carboxy-terminal propeptide of type I procollagen (PICP) in the culture medium, cell fractions, and serum samples, as measures of type III and type I collagen synthesis, respectively. The radioimmunoassays used are specific and showed no cross reactions with each other. $^{67}$

\section{RNA ANALYSIS}

Total RNA was isolated from confluent fibroblast cultures of a healthy control and the patient's cell line. ${ }^{8}$ For northern blot hybridisations, cDNA probes for the genes of the proa 1 chain of type III procollagen, ${ }^{9}$ the proa 2 chain of type I procollagen, ${ }^{10}$ elastin, ${ }^{11}$ and $\beta$ actin (Clontech Laboratories, CA) were used separately. The filter was exposed overnight to the radiographic film after being hybridised with the probe for $\beta$ actin and for seven days after the hybridisations with the probes for type I and III collagen and elastin.

\section{HISTOLOGICAL STUDIES}

Skin biopsies taken from the volar surface of the left forearm at the age of 2 years 4 months as well as the tissue samples collected at

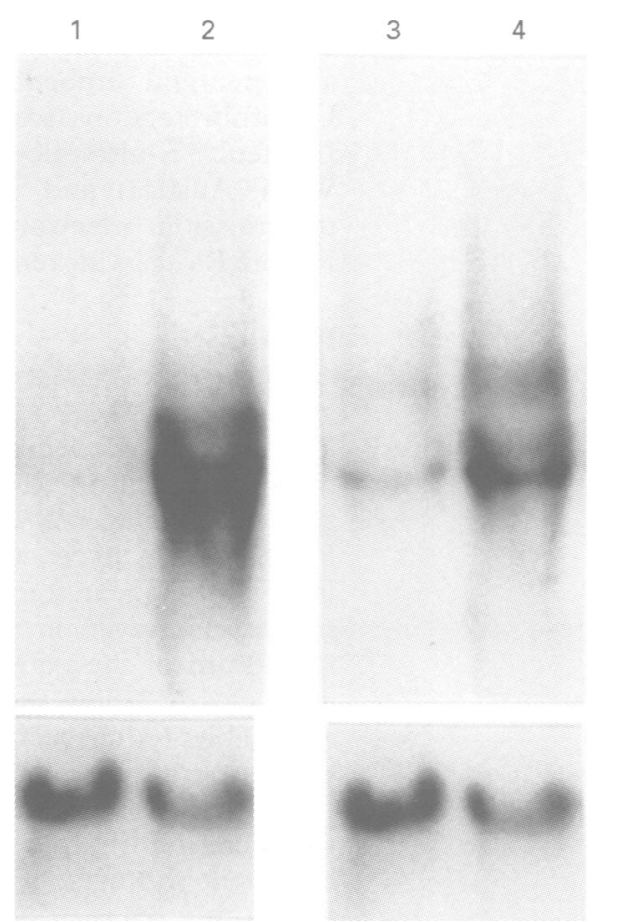

Figure 3 Northern blot analysis of RNAs. Lanes 1 and 3 contain $27 \mu \mathrm{g}$ of total RNA isolated from the patient's cultured skin fibroblasts; $30 \mu \mathrm{g}$ of total RNA isolated from control fibroblasts were run in lanes 2 and 4 . Lanes 1 and 2 were hybridised with a probe corresponding to human proa1 (III) collagen $m R N A s$, and lanes 3 and 4 with a probe corresponding to human proa2(I) collagen mRNAs. The bands below represent control hybridisations with a probe corresponding to $\beta$ actin $m R N A s$. 


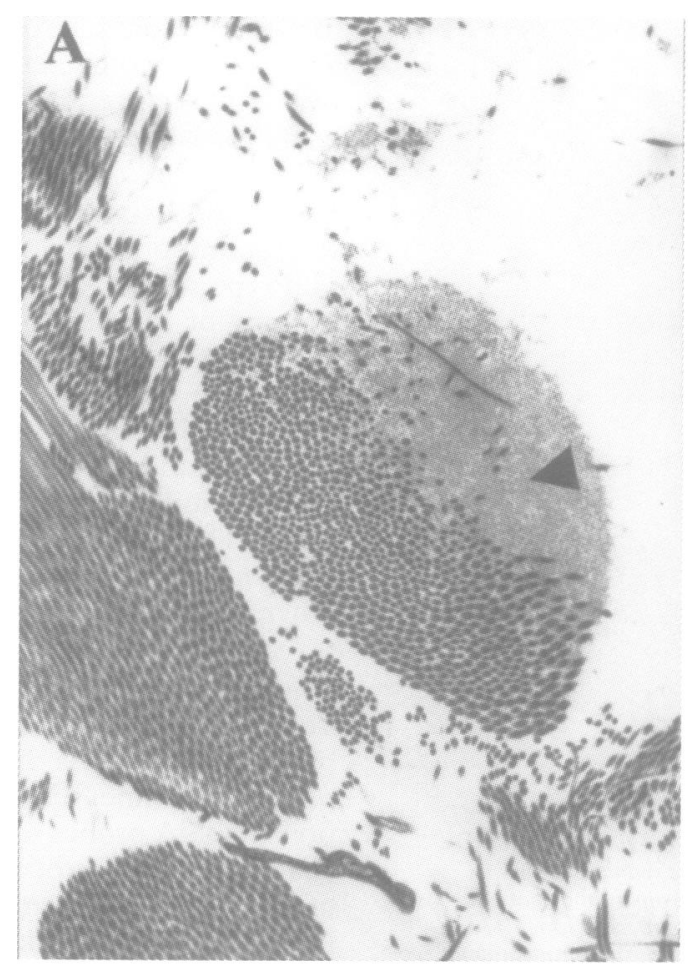

Figure 4 Electron micrograph of the dermis of forearm skin. (A) The collagen fibres are accompanied by thin,

disorganised fibres (arrowhead) without adjacent fibroblasts. (B) Elastic fibres (star) seem to be composed of tubular fibrils, but the core of amorphous material seems to be lacking.

necropsy were fixed in buffered $4 \%$ formalin, embedded in paraffin, and stained with haematoxylin-eosin and Alcian blue-periodic acid-Schiff methods. Elastic fibres were visualised with the Verhoff stain.

For electron microscopy, tissue samples from skin and aorta were first fixed in $2.5 \%$ glutaraldehyde, then postfixed in $1 \% \mathrm{OsO}_{4}$ in $0.1 \mathrm{~mol} / 1$ phosphate buffer, dehydrated in acetone, and embedded in Epon LX 112. Ultrathin sections were cut with a Reichert Ultracut E-ultramicrotome (Reichert-Jung, Wien, Austria) and examined in a $410 \mathrm{LS}$ transmission electron microscope (Phillips Export BV, Eindhoven, The Netherlands).

\section{Results}

PRODUCTION OF TYPES I AND III PROCOLLAGEN IN VIVO AND IN VITRO

The circulating concentrations of two propeptides set free during the synthesis of type I and type III collagens, abbreviated PICP and PIIINP, respectively, were within the reference intervals for age in the mother and father of the patient. However, in the repeated analyses of the serum of the patient both concentrations were clearly below the reference intervals for age (table 1), suggesting a defect that affects both these major collagens.

The same methods were used for quantifying the secretion of types I and III procollagen in confluent cultures of fibroblasts. In the patient's cultures the concentration of PIIINP in the culture medium was less than a third and that of PICP less than a fifth of the corresponding concentrations in the control culture (table 2 ). To characterise the procollagens produced by the cells further, confluent cultures were labelled with radioactive proline and the newly formed radioactive proteins studied by gel

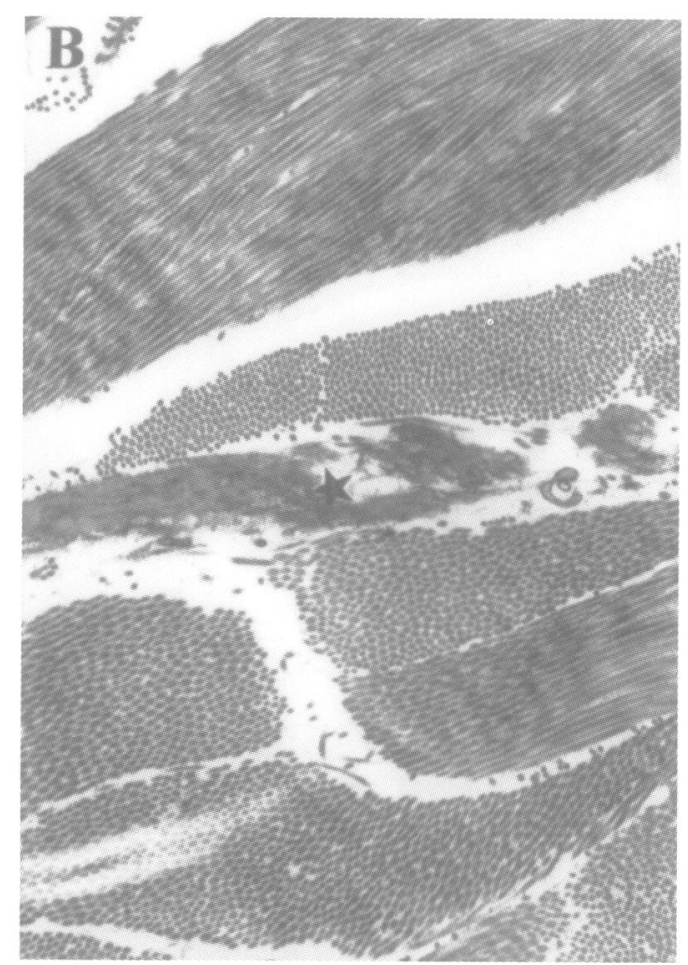

electrophoresis and immunoprecipitation with specific antibody against type III procollagen (fig 2). The medium and cell layer fractions of a control culture of adult fibroblasts contained the characteristic bands of type I and type III procollagens and collagens, ${ }^{3}$ whereas the polypeptide patterns of the patient's culture were clearly different. In the control, the bands related to type III procollagen could be easily visualised by immunoprecipitation with antiPIIINP antibodies (fig 2, lane 5), whereas nothing could be seen in the patient's medium treated identically (fig 2, lane 6). All these results agree with an almost total lack of type III procollagen production, but type I procollagen production is also defective.

\section{MESSENGER RNA ANALYSIS}

Northern blot hybridisation of the RNA produced by the patient's fibroblasts showed the two transcripts corresponding to the proa 2 chain of type I procollagen in amounts remarkably lower than those produced by control cells. The two type III procollagen mRNA bands were barely visible in the patient's samples. Hybridisation with the elastin probe showed that the amount of elastin mRNA was also decreased in the patient's fibroblasts (data not shown). $\beta$ actin mRNA was analysed as an internal control and was clearly visible both in the patient and control samples. The sizes of all the mRNA species studied appeared normal (fig 3).

HISTOLOGICAL FINDINGS

In skin biopsies, the dermis appeared thinner and the elastic fibres fragmented and less numerous than in age matched control skin. Ultrastructurally, the elastic fibres seemed to lack the amorphous body normally observed, 
Table 3 Comparison of the patient with Ehlers-Danlos syndrome type IV, geroderma osteodysplastica, and De Barsy syndrome

\begin{tabular}{lllll}
\hline & Patient & EDS IV & GO & De Barsy \\
\hline Severe growth retardation & + & - & + & + \\
Mental retardation & - & - & - & + \\
Progressive microcephaly & - & - & - & + \\
Cataracts/corneal opacities & + & - & - & + \\
Hypermobility of small joints & + & + & + & + \\
Hypermobility of large joints & + & - & - & - \\
Generalised osteoporosis & + & - & + & + \\
Hernias & + & + & - & + \\
Thin translucent skin/visible veins & + & + & + & + \\
Loose skin & + & - & + & + \\
Vascular manifestations & + & + & - & - \\
Inheritance & $?$ & AD & AR & AR \\
Premature ageing & + & - & + & + \\
Abnormal elastic fibres & + & - & $+/-$ & + \\
Cartoon dwarf-like facial appearance & + & - & + & - \\
Verified type I collagen defect & + & - & - & - \\
Verified type III collagen defect & + & + & - & - \\
\hline
\end{tabular}

$\mathrm{AR}=$ autosomal recessive inheritance, $\mathrm{AD}=$ autosomal dominant inheritance + indicates positive findings and - indicates both negative findings and findings not known.

and appeared fragmented and overstained (fig $4 \mathrm{~A})$. In some locations, the collagen fibres were in close contact with a delicate meshwork of apparently disorganised fibres (fig 4B). A striking feature of the dermis was the sparseness, or even lack, of fibroblast cells.

In the biopsy taken from the ascending aorta at necropsy, collagen fibres were of normal appearance, but there was more material staining with Alcian blue, suggesting increased amounts of proteoglycan, between the collagen fibres. The elastic fibres appeared fragmented but evenly stained.

\section{Discussion}

Our patient suffered from a disorder characterised by extreme hypermobility of the joints, inability to move, laxity of the skin, severe growth retardation, cataracts, local osteoporosis, and progressive spinal stenosis. He had several features in common with three inherited diseases affecting connective tissue, growth, and ageing (table 3): subtype IV of Ehlers-Danlos syndrome (EDS), geroderma osteodysplastica (GO), and De Barsy syndrome. A verified type III collagen defect is characteristic of EDS IV, ${ }^{12}$ but our patient had no bruising or scarring tendencies. His growth retardation and poor development were also far too severe for a typical EDS IV patient, and the type I collagen defect observed is definitely not present in this disease (table 3).

Some of the findings are similar to cardinal features described in patients with GO (table 3). ${ }^{13}$ In particular, his facial appearance with the small, slightly dysmorphic left ear and his close resemblance to the dwarfs of Walt Disney's Snow White are remarkably similar to the proband (III.3) of the second family in the report of Hunter $e t a^{14}$ on GO. However, severe generalised osteoporosis has been one of the main features in other published GO patients. In our patient, osteoporosis became evident only later and increased with age and long immobilisation. Also, the facial skin was not wrinkled, but rather had a tight appearance. As far as we know, all geroderma patients have eventually been ambulatory and had normal speech development, unlike our patient.
Both abnormal ${ }^{13}{ }^{15}$ and normal ${ }^{16}$ findings in skin biopsies have been reported in GO. The fragmentation of the elastic fibres seen in skin in GO as well as in our patient is not necessarily a specific finding. To our knowledge, no abnormal biochemical collagen findings have been reported in this disorder.

Degeneration of the elastic tissue in skin and cornea, together with retarded growth and mental retardation, are features of De Barsy syndrome. ${ }^{17}$ Mental retardation is severe in most patients and some patients never learn to speak, but intelligence can also be only mildly impaired, ${ }^{18}$ or even normal. The patients show athetoid and choreiform movements and peculiar grimacing. With the exception of the report by Hoefnagel $e t a l,{ }^{19}$ degeneration of collagenous or elastic fibres has usually been observed when a skin biopsy has been performed. The postnatal development of microcephaly, typical of De Barsy patients, was not found in our patient, but occasional peculiar grimacing was seen.

Our patient's fibroblasts showed clearly diminished production of mRNA for both type I and III procollagen (fig 3). Also the production of the corresponding proteins was decreased, suggesting possible defects at the gene level. Practically no newly synthesised type III procollagen could be detected in the cell cultures, indicating minimal production of this protein (fig 2). The control samples were from adult skin, but this should not affect the results as collagen synthesis is at its most active in infancy and early childhood. ${ }^{20}$

The serum findings agree with the in vitro results on type III procollagen production (table 1). The circulating concentration of PIIINP, a propeptide released from type III procollagen, was 50 to $80 \%$ lower than normal for his age group. A similar decrease was seen in the concentration of PICP, a propeptide derived from type I collagen synthesis. The parents showed concentrations within the adult reference intervals in both analyses (table 1).

The similarly decreased production rate of two different collagen types was a surprising finding. The three genes for type I (COL1A1 and COL1A2) and type III (COL3A1) procollagens are all located on different chromosomes. ${ }^{21}$ However, it is highly improbable that there would be mutations in all or even two of these genes in the same patient. We did not detect any gross structural defects either in the proteins or in the corresponding mRNAs. In fibroblast cells, the expression of type I and type III procollagen is tightly coordinated, ${ }^{22}$ whereas in mature osteoblasts only type I collagen is produced. Neither of these regulatory mechanisms is completely known at the molecular level.

Our patient could in principle have a defective gene for some regulatory protein involved in the coordinated regulation of type I and type III collagen expression. As a genetic defect this should have led to a combined clinical picture of severe osteoporosis or osteogenesis imperfecta and EDS IV, being most probably early lethal. However, our patient does not fulfil the criteria of osteogenesis imperfecta or severe 
osteoporosis. Radiographic findings in the patient did not show the changes of osteogenesis imperfecta. The immunological and biochemical methods showed reduced synthesis of type I procollagen, but also an extensive lack of type III procollagen, which is not typical of osteogenesis imperfecta. In osteogenesis imperfecta, procollagen type III synthesis is not affected.

The clinical picture and the collagen defect of the patient resemble the process of accelerated senescence of the fibroblasts and other cells of mesenchymal origin, as the capacity for collagen turnover tends to become lower. This is indirectly suggested in our patient by an almost normal collagen content of the skin together with a minimal amount of newly synthesised collagen.

Genetic counselling for this family is difficult as both autosomal recessive and dominant inheritance are found in the disorders discussed here. Being the offspring of clinically healthy parents with biochemically normal collagen findings, our patient's mutation is most probably new if dominantly inherited. However, the other modes of inheritance cannot be excluded either. The radioimmunoassays used in this study were clinically useful and easy for diagnosing the major changes involving the type I and III collagen metabolism of our patient. If the collagen defects detected in this child could be measured directly in amniotic fluid as in osteogenesis imperfecta (Kauppila et $a l$, unpublished data), prenatal diagnosis using specific radioimmunoassays could be offered to this family in the future.

We gratefully acknowledge the expert technical assistance of Ms Kristiina Apajalahti and Ms Outi Laurila.

1 Beighton P. McKusick's heritable disorders of connective tissue. St Louis: Mosby, 1993.

2 Jukkola A, Risteli J, Niemelä O, Risteli L. Incorporation of sulphate into type III procollagen by cultured human fibroblasts. Identification of tyrosine O-sulphate. Eur $\mathcal{F} B i_{0}$ chem 1986;154:219-24.

3 Jukkola A, Risteli J, Risteli L. Effect of dextran on synthesis, secretion and deposition of type III procollagen in cultured human fibroblasts. Biochem $\mathcal{F}$ 1991;279:49-54.
4 Cooper AR, Kurkinen M, Taylor A, Hogan BLM. Studies on the biosynthesis of laminin by murine parietal endoderm cells. Eur $\mathcal{F}$ Biochem 1981;119:189-97.

5 Laemmli UK. Cleavage of structural proteins during the assembly of the head of bacteriophage T4. Nature 1970;277:680-5.

6 Risteli J, Niemi S, Trivedi P, Mäentausta O, Mowat AP, Risteli L. Rapid equilibrium radioimmunoassay for the aminoterminal propeptide of human type III procollagen. Clin Chem 1988;34:715-18.

7 Melkko J, Niemi S, Risteli L, Risteli J. Radioimmunoassay of the carboxyterminal propeptide of human type I procollagen. Clin Chem 1990;36:1328-32.

8 Chirgwin IM, Przybyla AE, MacDonald RJ, Rutter WJ. Isolation of biologically active ribonucleic acid from sources enriched in ribonuclease. Biochemistry 1979;18:5294-9.

9 Loidl HR, Brinker JM, May M, et al. Molecular cloning and carboxylpropeptide analysis of human type III procollagen. Nucleic Acids Res 1984;12:9383-94.

10 Bernard MP, Myers JC, Chu ML, Ramirez F, Eikenberry EF, Prockop DJ. Structure of a cDNA for the proa 2 chain of human type I procollagen. Comparison with chick cDNA for proa2(I) identifies structurally conserved features of the protein and the gene. Biochemistry 1983;22: features of

11 Fazio MJ, Olsen DR, Eunkyung AK, et al. Cloning of full-length elastin cDNA library: further elucidation of alternative splicing utilizing exon-specific oligonucleotides. Invest Dermatol 1988; 91:458-64.

12 Pope FM, Nicholls AC, Narcisi P, et al. Type III collagen mutations in Ehlers Danlos syndrome type IV and other related disorders. Clin Exp Dermatol 1988;13:285-302.

13 Lisker R, Hernandez A, Martinez-Lavin M, et al. Gerodermia osteodysplastica hereditaria: report of three affected brothers and literature review. Am $\mathcal{F}$ Med Genet 1979;3: 389-95.

14 Hunter AGW, Martsolf JT, Baker CG, Reed MH. Geroderma osteodysplastica. A report of two affected Geroderma osteodysplastica. A rep
families. Hum Genet 1978;40:311-24.

15 Klein D, Bamatter F, Franceschetti A, Boreaux G, Brocher $J E W$, Holenstein $P$. Une affection liée au sexe: la gérodermie ostéodysplasique héréditaire $(20$ ans d'observation). Rev Oto-Neuro-Ophtalmol 1968;40:415-21.

16 Suter H, Tönz O, Scharli A. Geroderma osteodysplastica heriditaria (GOH) in a girl. In: Papadatos CJ, Bartsocas CS, eds. Skeletal dysplasias. New York: Alan R Liss, 1982:327-9.

17 De Barsy AM, Moens E, Dierckx L. Dwarfism, oligophrenia and degeneration of the elastic tissue in skin and cornea. A new syndrome? Helv Paediatr Acta 1968;23:305-13.

18 Kunze J, Majewski F, Montgomery P, Hockey A, Karkut I, Riebel T. De Barsy syndrome-an autosomal recessive, progeroid syndrome. Eur $\mathcal{F}$ Pediatr 1985;144:348-54.

19 Hoefnagel D, Pomeroy J, Wurster D, Saxon A. Congenital athetosis, mental deficiency, dwarfism and laxity of skin and ligaments. Helv Paediatr Acta 1971;26:397-402.

20 Trivedi P, Risteli J, Risteli L, Hindmarsh PC, Brook CGD, Mowat AP. Serum concentrations of type I and III procollagen propeptides as biochemical markers of growth in infants and children with growth disorders. Pediatr Res 1991;30:276-80.

21 Vuorio E, de Crombrugghe D. The family of collagen genes. Annu Rev Biochem 1990;59:837-72.

22 Vuorio T, Mäkelä JK, Kähäri VM, Vuorio E. Coordinated regulation of type I and type III collagen production and $\mathrm{mRNA}$ levels of proal (I) and proa2(I) collagen in cultured morphea fibroblasts. Arch Dermatol Res 1987;279:154-60. 